\section{Medication Therapy Management and Collaborative Drug Therapy Management}

With the current push for health care reform, many are asking how ongoing government programs like Medicare Part D are performing. The Medicare Modernization Act of 2003 established the requirement that Medicare Part D prescription drug plans (PDPs) and Medicare Advantage prescription drug plans (MA-PDs) provide medication therapy management programs (MTMP) using pharmacists or other qualified health care providers as part of their benefits. ${ }^{1}$ In general, medication management services encompass care beyond traditional medication counseling. MTM services are designed to maximize the benefits of prescribed medication regimens, increase medication adherence, and reduce the risk of adverse drug events and drug interactions. ${ }^{2}$ In an article by Spooner (2007), he suggested that at the 1-year mark, it was time to start evaluating MTMP services. ${ }^{3}$ Spooner recommended that evaluations focus on the following 2 questions: "are we correctly identifying patients who would benefit the most and has the MTMP been successful in achieving established goals." 3 According to the Medication Therapy Management in Pharmacy Practice: Core Elements of an MTM Service Model Version 2.0, MTM programs should also be designed "to improve collaboration among pharmacists, physicians, and other health care professionals, enhance communication between patients and their health care team; and optimize medication use for improved patient outcomes."

In a recently published article in JMCP, Cook and MburiaMwalili discussed Medicare Part D policy changes that have affected pharmacists working in MTMP. They described how the availability of the National Provider Identification (NPI) numbers and pharmacy-specific Current Procedural Terminology (CPT) codes have facilitated the implementation of MTMP by allowing pharmacists to seek reimbursement for their services. ${ }^{5}$ The authors suggest that with a wide range of services being provided including mailed information, telephone consultations, as well as in-person appointments, large chain community pharmacies may be the best settings for the provision of MTM because of previously developed billing infrastructure and in-store clinics with prior trained clinical staff. However, Cook and Mburia-Mwalili acknowledge that even with a collaborative approach of pharmacist and prescriber, community pharmacy chains would not likely manage more serious chronic conditions. We foresee collaborative drug therapy management programs as a way to gather data for outcomes of improvements of these chronic conditions.

\section{Collaborative Drug Therapy Management}

CDTM refers to collaborative practice agreements between pharmacists and prescribers in which pharmacists are enabled to initiate, modify, or continue drug therapy for specific patients as defined in a written guideline or protocol. ${ }^{6}$ CDTM is being used in the following areas: immunizations, asthma therapy, dyslipidemia therapy, warfarin anticoagulation therapy, diabetic therapy, smoking cessation therapy, and flu/antiviral therapy. Currently, 46 states in the U.S. have authorized CDTM agreements between pharmacists and physicians, ${ }^{7}$ including most recently Massachusetts ${ }^{8}$ and Rhode Island. ${ }^{9}$ Of the 4 states that do not have CDTM programs (Alabama, Oklahoma, Maine, and New York), pharmacists in New York are lobbying to implement a CDTM bill (S.3292/A.6448). ${ }^{10}$

Kaiser Permanente of Georgia (KPGA) health plan is a nonprofit health maintenance organization that provides integrated health care services to more than 250,000 members at 15 medical offices in the Atlanta metropolitan area. At KPGA, clinical pharmacists are part of a collaborative team that provides CDTM and MTM for patients diagnosed with dyslipidemia, diabetes, hypertension, or coronary artery disease as defined in protocols developed by clinical pharmacists and primary care physicians (PCPs). A patient-specific protocol is valid for 2 years unless the patient changes physicians or disenrolls from the KPGA health plan. The CDTM protocols include algorithms for specific drug therapy modifications, dosing ranges, and monitoring information for insulin management, hypertension management, diabetes management, lipid management, lab monitoring, and aspirin therapy initiation.

Patients are enrolled into these programs through direct referral by PCPs or via administrative queries of a Web-based electronic database. When a protocol is signed by a PCP, all current patients of that PCP are authorized to be managed under the protocol as well as future patients that are individually referred to clinical pharmacists by that PCP. The collaborative protocols with KPGA PCPs allow the clinical pharmacists to confer with patients and other members of the health care team to initiate medications, order laboratory tests, and adjust dosages on the basis of lab results or reported side effects, as well as make formulary conversions.

In addition to the use of collaborative protocols, KPGA providers, patients, and staff also rely heavily upon electronic medical records (EMRs). The database used for referrals is integrated with the KPGA EMR and the collective data are used to identify patients who are due for laboratory tests, those who are diagnosed with specific chronic diseases and have not met their goals, have not seen their PCPs in more than 1 year and Medicare Part D patients eligible for enrollment in the MTMP. EMRs offer access to a complete patient history, including, but not limited to procedures, medication recommendations, and existing medical conditions. At KPGA, the clinical pharmacists and the health care team use EMRs to coordinate patient care at each point of service, including office visits, laboratory services, prescription services, hospitalizations, phone consultations, and online services. Patients enrolled in the KPGA health plan have access to view online portions of their medical record and information about their office visits. The Web-based portal also allows 
patients to safely and securely send messages to their providers, view lab results, schedule appointments, and order medications for pick-up or delivery. The use of this innovative information technology may potentially reduce fragmentation of medical care as it increases collaboration among providers.

\section{MTMP at KPGA}

In 2009, the Centers for Medicare and Medicaid Services (CMS) reported that in 2008 there were 712 active Part D approved MTM programs, 609 MA-PD and 103 PDP. ${ }^{11}$ Criteria for these programs vary, however, targeted beneficiaries must have multiple chronic diseases to be enrolled. The top 4 chronic conditions frequently targeted by MTMPs in 2008 were diabetes, heart failure, hypertension, and dyslipidemia. Enrollees must also be taking multiple Medicare Part D drugs which are likely to incur annual costs of $\$ 4,000$ or greater. ${ }^{11}$ The minimum number of covered Medicare Part D drugs to determine MTMP eligibility varied among MTMPs in 2008, ranging from as few as 2 to as many as 15; most programs required a minimum of 5 to 8 medications (62.4\%) per beneficiary for MTMP eligibility. ${ }^{11}$

In the second quarter of 2009, the clinical pharmacists at KPGA began monitoring MTM patients under the collaborative protocol. The KPGA MTMP identifies and invites patients that are likely to incur annual drug costs greater than or equal to $\$ 4000$, are taking at least 5 chronic or maintenance medications covered by Medicare Part D, and have at least 2 of the 5 following chronic conditions: asthma, diabetes, chronic kidney disease, hypertension, and coronary artery disease. Patient participation in the KPGA MTMP is voluntary, and MTM clinical pharmacists attempt to provide services to all MTMP-eligible members. Initially, welcome letters are mailed, and patients are later contacted by telephone and invited to participate in the MTMP. If a patient is unable to be reached by telephone after 3 attempts, a letter is mailed requesting his/her response by a specified date to become enrolled in the program. Failure of the patient to respond by the specified date will indicate the member's decision not to participate in the MTMP. Eligible members may opt out at any time by contacting their MTM clinical pharmacists. MTMP enrollment has grown from 101 patients in 2009 Q1 to 270 patients in 2009 Q3, and currently 330 (44\%) of 750 MTMPeligible members are enrolled in the KPGA MTMP.

After enrollment, MTM clinical pharmacists perform an initial assessment to validate the complete medication profile and the following target areas: patient compliance, drug therapy duplication, potential drug interactions, potential cost-effective alternatives, and drug therapy appropriateness. Next, MTM patients have their individualized therapeutic goals defined. After the initial patient assessment, the MTM pharmacist will conduct follow-up telephone encounters with the patient at least quarterly or more frequently if necessary, to assess progress toward treatment goals. Patient-specific education and instruction regarding medication use is provided, as appropriate, during each encounter.

Going forward, CDTM protocols may be used to define outcome measures such as low-density lipoprotein (LDL) goals and hemoglobin Alc goals, and identify the patients who are due for laboratory monitoring. Currently, preliminary outcomes data are being collected on the use of high-risk medications in the elderly to monitor for safety.

\section{Conclusion}

Pharmacists play a central role in management of medication therapy, particularly for patients with chronic conditions, and CDTM can enhance that role. ${ }^{12-14}$ The EMR in our health system allows for seamless collaboration and the sharing of information among providers and their patients. With the use of a Web portal for access to the electronic database and the list of patients enrolled in CDTM protocols, we are able to identify Medicare patients who are monitored by clinical pharmacists at KPGA. The CDTM protocols and agreements between clinical pharmacists and PCPs facilitate the delivery of MTMP services in our health system.

\section{Stephanie Roberts, PharmD, BCPS}

Clinical Pharmacy Specialist

Kaiser Permanente

Stephanie.Roberts@kp.org

Rachel Gainsbrugh, PharmD

Clinical Pharmacy Specialist

Kaiser Permanente

Rachel.D.Gainsbrugh@kp.org

\section{DISCLOSURES}

The authors disclosed no conflicts of interest in this subject. Both authors work in delivering MTM services.

The authors wish to acknowledge Scott Cooper, PharmD, Clinical Pharmacy Assistant Manager, Kaiser Permanente, for his assistance with this Letter.

\section{REFERENCES}

1. Public Law 108-173. The Medicare Prescription Drug, Improvement, and Modernization Act of 2003. December 8, 2003. Available at: http://www. treas.gov/offices/public-affairs/hsa/pdf/pl108-173.pdf. Accessed November 22, 2009 .

2. Rovers J, Currie J, Hagel H, et al. Re-engineering the pharmacy layout. In: A Practical Guide to Pharmaceutical Care. 2nd ed Washington DC: American Pharmacists Association; 2003.

3. Spooner JJ. Medication therapy management programs: when will the outcomes come out? J Manag Care Pharm. 2007;13(3):276-77. Available at: http://www.amcp.org/data/jmcp/276-77.pdf. 
4. American Pharmacists Association and the National Association of Chain Drug Stores Foundation. Medication therapy management in pharmacy practice: core elements of an MTM service model (version 2.0). J Am Pharm Assoc. 2008;48:341-53. Available at: https://www.aphanet.org/AM/Template. $\mathrm{cfm} ?$ Section=Home\&Template $=/ \mathrm{CM} /$ ContentDisplay. $\mathrm{cfm} \&$ ContentID $=16857$. Accessed November 23, 2009.

5. Cook DM, Mburia-Mwalili A. Medication therapy management favors large pharmacy chains and creates potential conflicts of interest. J Manag Care Pharm. 2009;15(6):495-500. Available at: http://www.amcp.org/data/ jmcp/495-500.pdf.

6. Hammond RW, Schwartz AH, Campbell MJ, et al. American College of Clinical Pharmacy. ACCP Position Statement: Collaborative drug therapy management by pharmacists-2003. Pharmacotherapy. 2003;23(9):121025. Available at: http://www.accp.com/docs/positions/positionStatements/ pos2309.pdf. Accessed November 26, 2009.

7. Alliance for Pharmaceutical Care. Collaborative drug therapy management: a coordinated approach to patient care. Available at: http://www.ashp. org/s_ashp/docs/files/about/CDTM.doc. Accessed December 5, 2009.

8. Massachusetts College of Pharmacy and Health Sciences. Gov. Patrick signs landmark collaborative drug therapy management bill into law. January 16, 2009. Available at: http://www.mcphs.edu/news_and_events/ press_releases/2009/2009.01.16.gov_patrick_signs_drug_therapy_bill.html. Accessed December 5, 2009.

9. State of Rhode Island and Providence Plantations, Department of Health. Rules and regulations pertaining to pharmacists, pharmacies and manufacturers, wholesales and distributors [R5-19.1-PHAR], Section 25, collaborative pharmacy practice. Available at: http://www2.sec.state.ri.us/dar/regdocs/ released/pdf/DOH/5763.pdf. Accessed December 5, 2009.
10. New York State Assembly. An act to amend the education law, in relation to authorizing pharmacists to perform collaborative drug therapy management with physicians or nurse practitioners in certain settings and providing for the repeal of such provisions upon expiration thereof, S.3292/A.6448 11lth Cong, lst Session (2009). Available at: http://assembly.state.ny.us/ leg/?bn=A06848. Accessed November 22, 2009.

11. Centers for Medicare \& Medicaid Services. Medicare Part D Medication Therapy Management (MTM) Programs: 2009 Fact Sheet. Available at: http://www.cms.hhs.gov/PrescriptionDrugCovContra/Downloads/ MTMFactSheet.pdf. Accessed November 22, 2009.

12. Horberg MA, Hurley LB, Silverberg MJ, Kinsman CJ, Quesenberry CP. Effect of clinical pharmacists on utilization of and clinical response to antiretroviral therapy. J Acquir Immune Defic Syndr. 2007:44(5):531-39. Available at: http://journals.lww.com/jaids/Fulltext/2007/04150/Effect_of_Clinical_ Pharmacists_on_Utilization_of.6.aspx. Accessed November 23, 2009.

13. Hunt JS, Siemienczuk J, Pape G, et al. A randomized controlled trial of team-based care: impact of physician-pharmacist collaboration on uncontrolled hypertension. J Gen Intern Med. 2008;23(12):1966-72. Available at: http://www.ncbi.nlm.nih.gov/pmc/articles/PMC2596500/pdf/11606_2008_ Article_791.pdf. Accessed November 24, 2009.

14. Anaya JP, Rivera JO, Lawson K, Garcia J, Luna J Jr, Ortiz M. Evaluation of pharmacist-managed diabetes mellitus under a collaborative drug therapy agreement. Am J Health Syst Pharm. 2008;65(19):1841-45. Available at: http:// www.ncbi.nlm.nih.gov/pubmed/18796426?itool=EntrezSystem2.PEntrez. Pubmed.Pubmed_ResultsPanel.Pubmed_RVDocSum\&ordinalpos=15. Accessed November 27, 2009. 